\title{
Orientações motivacionais de alunos do curso de biblioteconomia
}

\author{
Adriana Rosecler \\ Sueli Édi Rufini Guimarães
}

\section{Resumo}

A motivação é um importante problema de investigação, pois tem implicações diretas na qualidade do envolvimento do aluno com a aprendizagem. Nesta pesquisa, analisaram-se as orientações motivacionais de alunos do curso de Biblioteconomia, de uma universidade pública do norte do Paraná, a partir da identificação do tipo de motivação, das estratégias de aprendizagem utilizadas, da percepção de desempenho acadêmico e da Perspectiva de Tempo Futuro. Participaram do estudo 143 alunos das quatro séries do curso. Os dados da pesquisa foram coletados por meio de um questionário de autorrelato, contendo 80 questões do tipo escala likert, elaboradas e analisadas de acordo com a Teoria da Autodeterminação e a Perspectiva de Tempo Futuro. Os resultados indicaram uma tendência para os tipos mais autônomos de motivação, o estabelecimento de metas a serem alcançadas no futuro e a percepção das atividades acadêmicas do presente como instrumentais para o alcance dessas metas futuras.

Palavras-Chave: Motivação, aprendizagem, rendimento escolar.

\section{Motivational orientations of library course students}

\begin{abstract}
Motivation is an important research problem because it has direct implications on the quality of student engagement with learning. In this study, we analyzed the motivational orientations of students of librarianship, a public university in the north of Paraná, from the identification of the type of motivation, learning strategies used, the perception of academic performance and Time Perspective future. Study participants were 143 students in four grades of course. The survey data were collected through a self-report questionnaire containing 80 likert-type questions, compiled and analyzed in accordance with the self-determination theory and the future time perspective theory. The results indicated a trend towards more autonomous types of motivation, setting goals to be achieved in the future and the perception of the academic activities of this as instrumental to achieving these future goals.
\end{abstract}

Key-words: Motivation, learning, academic achievement.

\section{Orientaciones de motivación de alumnos de la facultad de biblioteconomía}

\section{Resumen}

La motivación es un importante problema de investigación, ya que tiene implicaciones directas en la calidad de la participación del alumno con el aprendizaje. En este estudio, se analizaron las orientaciones de la motivación de alumnos de la facultad de Biblioteconomía, de una universidad pública del norte de Paraná, a partir de la identificación del tipo de motivación, de las estrategias de aprendizaje utilizadas, de la percepción de rendimiento académico y de la Perspectiva de Tiempo Futuro. Participaron del estudio 143 alumnos de los cuatro años de la facultad. Los datos de la investigación se recogieron por medio de cuestionario de auto-relato, conteniendo 80 preguntas del tipo escala likert, elaboradas y analizadas de acuerdo con la Teoría de la Autodeterminación y la Perspectiva de Tiempo Futuro. Los resultados indicaron una tendencia para los tipos más autónomos de motivación, el establecimiento de objetivos que deben alcanzarse en el futuro y la percepción de las actividades académicas del presente como instrumentales para lograr esas metas futuras.

Palabras-Clave: Motivación, aprendizaje, rendimiento escolar. 


\section{Introdução}

No Brasil, já se tem uma pequena coletânea de estudos sobre a motivação de alunos. Boruchovitch e Bzuneck (2010) apresentam um panorama dos estudos brasileiros realizados a partir dos anos 90 . Apesar de ainda em número reduzido, os resultados das pesquisas brasileiras, desenvolvidas na perspectiva de diversas teorias, oferecem algumas pistas que podem colaborar para a atuação dos professores em sala de aula.

Entre as teorias que fornecem suporte para a compreensão da motivação, está a Teoria da Autodeterminação (TAD), a qual foi a abordagem que embasou o presente estudo. Proposta por Deci e Ryan na década de 70 do século $X X$, a teoria focaliza as tendências naturais humanas para o crescimento, desenvolvimento e bem-estar no processo de interação com as condições socioculturais (Deci \& Ryan, 2000, 2008; Reeve, Deci, \& Ryan, 2004). Nessa perspectiva, o ser humano move-se para satisfazer três necessidades psicológicas básicas de autonomia, competência e pertencimento, consideradas como os nutrientes fundamentais para um relacionamento efetivo e saudável com o ambiente. A satisfação dessas necessidades parece ser essencial para fomentar a sensação de bem-estar e as propensões naturais para o crescimento e a integração do organismo. A TAD é atualmente considerada como uma perspectiva promissora para a compreensão acerca do envolvimento, persistência, desempenho e uso de estratégias de aprendizagem pelos estudantes no contexto escolar (Deci \& Ryan, 2008; Ryan \& Deci; 2000b).

Como resultado do aprofundamento teórico e do grande número de pesquisas empíricas realizadas na perspectiva da Teoria da Autodeterminação, foram elaboradas quatro subteorias - Teoria das Necessidades Básicas, Teoria da Avaliação Cognitiva, Teoria da Orientação de Causalidade e Teoria da Integração Organísmica, que objetivam entender, explicar e propor estratégias de intervenção, no contexto de interações sociais, potencializando condições para o desenvolvimento saudável e bem-estar. Nessa pesquisa, focalizou-se a Teoria da Integração Organísmica, que mostra como os alunos adquirem e internalizam os processos de regulação externos e evidencia a capacidade de gerar ações extrinsecamente motivadas, mas autodeterminadas. Isto é, as regulações externas podem ser internalizadas, transformando-se em internas.

A Teoria da Integração Organísmica propõe formas qualitativamente diferentes de motivação extrínseca, procurando descobrir os fatores do contexto que promovem ou dificultam a internalização dos valores e regulações externas. Esse processo de internalização é representado em um continuum de autodeterminação, sendo nele alocadas as diferentes modalidades de internalização. No ponto inicial está a desmotivação, definida como a ausência de intenção ou regulação para agir, a qual pode ser explicada pelo fato de o aluno não pretender trabalhar na atividade ou porque não vê nela razões para a sua execução.
Para os quatro tipos de motivação extrínseca, as regulações, valores ou comandos externos são gradualmente internalizados. Assim, a regulação externa é a forma menos autônoma de motivação extrínseca, em que o comportamento é instrumental para obter recompensas ou para evitar eventos desagradáveis. Na motivação extrínseca por regulação introjetada, embora o comportamento seja regulado internamente, o locus de causalidade ainda é externo, ou seja, as regulações estão na pessoa, mas não foram integradas ao self. É o exemplo do aluno que desenvolve suas atividades simplesmente para evitar que os outros o vejam como relapso ou para escapar de sentimentos de culpa e vergonha. Na motivação extrínseca por regulação identificada, o comportamento já é regulado de forma mais autônoma por empatia pela própria atividade a ser realizada, por seus valores ou exigências. A motivação extrínseca por regulação integrada representa a forma mais completa de internalização das demandas externas, havendo aceitação dos processos reguladores que, consequentemente, serão assimilados e integrados ao self. Nesse caso, o sucesso no processo de internalização é total e o aluno terá dificuldades em distinguir as demandas externas de seus próprios valores.

Finalmente, a motivação intrínseca, alocada no final do continuum, é o protótipo de motivação autodeterminada, havendo disposição natural e espontânea que impulsiona a pessoa a buscar novidades e desafios. Nesse caso, o envolvimento do aluno deve-se ao interesse pessoal e ao prazer associado à realização da atividade em si. Com base nesta perspectiva, os proponentes da teoria da integração organísmica (Ryan \& Deci 2000a, 2000b) superaram a visão dicotômica a respeito da motivação extrínseca e intrínseca presentes nas pesquisas anteriores aos anos 70 do século $\mathrm{XX}$. Em tais estudos, resultados positivos eram associados à motivação intrínseca e a motivação extrínseca, pouco explorada até então, relacionava-se com aprendizagem superficial, pouca persistência e baixo desempenho.

\section{Perspectiva de Tempo Futuro}

A Perspectiva de Tempo Futuro (PTF) refere-se ao grau e ao modo pelo qual o futuro cronológico de uma pessoa é integrado ao espaço de vida presente (Lens, Simons \& Dewitte, 2002). Resumidamente, podemos dizer que a Perspectiva de Tempo Futuro é uma antecipação no presente de metas futuras. Segundo Husman e Lens (1999) e Lens, Simons e Dewitte (2002), a consideração das metas futuras para entender o comportamento humano e a motivação já fazia parte dos estudos de Frank e Lewin, na década de 30. Nessa abordagem, a situação atual de uma pessoa incluía não apenas o seu presente, mas também o seu passado e o seu futuro.

Mais recentemente, as pesquisas sobre a Perspectiva de Tempo Futuro e suas implicações sobre a motivação humana têm sido desenvolvidas por Kauffman e Husman (2004), Mclnerney (2004), Miller e Brickman (2004), Phalet, Andriessen e Lens (2004), Simons, Dewitte e Lens (2004), 
Simons, Vansteenkiste, Lens e Lacante (2004), entre outros. Esses autores salientam a importância de se investigar e compreender como a concepção do futuro tem relação com o comportamento dos alunos no contexto acadêmico. Kauffman e Husman (2004), a partir de uma síntese das diversas pesquisas publicadas em uma edição especial do periódico Educational Psychology Review, concluíram que as concepções de futuro dos alunos têm uma influência real e significativa sobre as suas crenças e sobre a sua motivação para aprender, o que, por conseguinte, influencia positivamente o seu desempenho.

Simons e cols. (2004) analisaram a Perspectiva de Tempo Futuro e afirmaram que ela influencia a fixação de metas motivacionais e que há efeitos motivacionais relacionados às diferenças individuais na extensão da Perspectiva de Tempo Futuro. A respeito dessas diferenças individuais, Husman e Lens (1999) e Lens, Simons e Dewitte (2002) enfatizam que pessoas com Perspectiva de Tempo Futuro curta estabelecem suas metas num futuro próximo. Nesse caso, a distância temporal de futuro dessas pessoas é imediata ou próxima, por exemplo, fazer a tarefa logo para poder ir à piscina mais tarde. Em contrapartida, as pessoas com a Perspectiva de Tempo Futuro longa estabelecem metas a serem alcançadas num futuro mais distante e tendem a não ter dificuldades em se motivar para as atividades do presente, desde que estas tragam os resultados esperados, mesmo que em longo prazo. Além disso, estudos têm apontado que as diferenças individuais referentes à extensão da Perspectiva de Tempo Futuro incidem sobre a intensidade do esforço na motivação para aprender. Desse modo, as pessoas com uma Perspectiva de Tempo Futuro longa persistem mais em suas metas e sentem-se mais satisfeitas com os resultados de suas ações orientadas para as metas presentes ou imediatas (Lens, Simons, \& Dewitte, 2002; Simons e cols. 2004).

Ainda em relação à Perspectiva de Tempo Futuro, deve ser salientado o componente de instrumentalidade, que diz respeito à percepção da realização das atividades no presente como meio para atingir as metas futuras, ou seja, as atividades atuais são percebidas como instrumentais para a realização das metas futuras. De acordo com Malka e Covington (2005), o constructo de percepção de instrumentalidade, também denominado por alguns autores como motivação instrumental, teve um papel proeminente na clássica Teoria Expectativa-Valor de Atkinson. Atualmente, a percepção de instrumentalidade vem sendo estudada na abordagem da Perspectiva de Tempo Futuro, com ênfase na relação dos comportamentos em de sala de aula com as metas futuras. Ao se referir à instrumentalidade, Husman, Derryberry, Crowson e Lomax (2004) a descrevem como o resultado da conexão entre a valorização de uma tarefa atual e uma meta futura. Eles propuseram que a valorização de uma atividade e a expectativa de sucesso em relação a ela determinam a qualidade e a quantidade de esforço que o aluno dedicará durante a sua realização.

O presente estudo foi realizado com o objetivo de examinar as orientações motivacionais de alunos universitários. Buscou-se identificar o tipo de motivação, as estratégias de aprendizagem utilizadas, a percepção de desempenho e a Perspectiva de Tempo Futuro, assim como relacionar o tipo de motivação dos alunos com o uso de estratégias de aprendizagem e com a percepção de desempenho.

\section{Método}

\section{Participantes}

Os participantes desta pesquisa foram 143 alunos do curso de Biblioteconomia, do primeiro ao quarto ano, de uma universidade pública do norte paranaense, sendo 112 do gênero feminino e 31 do masculino. A faixa de idade variou de 19 a 36 anos de idade, com uma concentração de alunos na faixa de 20 a 25 anos.

\section{Instrumentos}

Foi utilizada uma escala composta de 80 questões. As 26 primeiras referiram-se às razões pelas quais os alunos vêm à universidade, denominada Escala de Motivação Acadêmica (EMA), versão brasileira elaborada e validada para uma amostra de 388 universitários (Guimarães \& Bzuneck, 2008). Nesse estudo, os autores extraíram itens da escala de Vallerand e cols. (1992) e elaboraram novos itens a partir da teoria. A análise fatorial exploratória revelou 7 fatores que explicaram $56,49 \%$ da variação dos dados e os índices de consistência interna dos itens que se agruparam em torno dos fatores variaram de 0,61 a 0,79.

Após a questão Por que venho à universidade?, os participantes deveriam assinalar, em escala likert de sete pontos, seu grau de concordância com afirmativas que indicavam desmotivação, 5 itens (Exemplo de item: Eu não sei por que devo vir à universidade), a motivação intrínseca, 4 itens (Exemplo de item: Porque para mim a universidade é um prazer) e os quatro tipos de motivação extrínseca, por regulação externa, 6 itens (Exemplo de item: Venho à universidade para conseguir o diploma), introjetada, 4 itens (Exemplo de item: Venho porque é isso que esperam de mim), identificada, 2 itens (Exemplo de item: Porque é isso que escolhi para mim) e integrada (Exemplo de item: Porque o acesso ao conhecimento se dá na universidade). Com a amostra do presente estudo, os itens elaborados para avaliar a motivação extrínseca por regulação integrada carregaram em mais de um fator ou não alcançaram a carga fatorial de 0,30, não sendo, portanto, analisados. A consistência interna dos itens das subescalas, avaliada a partir das respostas dos participantes na presente pesquisa, medida pelo alfa de Cronbach, resultou nos seguintes índices: desmotivação $(\alpha=0,74)$; motivação extrínseca por regulação externa $(\alpha=0,60)$; por regulação introjetada $(\alpha=0,71)$; por regulação integrada $(\alpha=0,61)$; e motivação intrínseca/regulação integrada. Na regulação identificada, não foi possível calcular o alfa, devido ao número reduzido de itens. 
A segunda parte do instrumento foi composta de 20 questões que avaliaram a Perspectiva de Tempo Futuro e as orientações dos alunos quanto ao seu futuro profissional também em escala likert de sete pontos, uma versão adaptada, para a presente pesquisa, do instrumento elaborado por Shell e Husman (2001). São exemplos de itens: "É importante ter metas para onde se quer estar em 5 ou 10 anos", "Ganhos imediatos são mais importantes do que a possibilidade de ganhos futuros". A análise da consistência interna dos 20 itens da escala da Perspectiva de Tempo Futuro apresentou um alfa de Cronbach de 0,79, indicando que a exclusão do item número 28 "O que faço hoje no curso terá pouco impacto no que acontecerá daqui a 10 anos" aumentaria esse índice para 0,81. Assim, a escala passou a ser composta de 19 itens.

A instrumentalidade e a valorização das atividades foram avaliadas por 7 questões em escala likert de 7 pontos, sendo que sua elaboração foi apoiada nos quatro tipos de instrumentalidade (baixa percepção de instrumentalidade e regulação externa; baixa percepção de instrumentalidade e regulação interna; alta percepção de instrumentalidade e regulação externa; alta percepção de instrumentalidade e regulação interna) apontados por Lens, Simons e Dewite (2002) e adaptados para o presente estudo. São exemplos de itens: "Eu faço as atividades desta disciplina simplesmente porque o professor exige" e "Quando estiver formado vou precisar realizar tarefas semelhantes às desenvolvidas nesta disciplina”. Para responder às questões, em cada série, foi solicitado aos alunos que pensassem em uma disciplina específica. A opção pela disciplina foi feita a priori pela proponente desta pesquisa, tendo como critérios a sua utilidade e relevância para a atuação profissional futura. Dentre as disciplinas constantes na grade curricular do curso, optouse pelas seguintes: $1^{\text {a }}$ série - "Representação Descritiva", $2^{\circ}$ série - "Formação e Desenvolvimento de Coleções", $3^{a}$ série - "Fontes e Redes de Informação Especializada" e $4^{a}$ série - "Projetos para Unidades de Informação". A escolha dessas disciplinas deveu-se ao fato de que essas estão entre as disciplinas consideradas essenciais para a formação do profissional bibliotecário.

Para a escala de avaliação da instrumentalidade, o índice de consistência interna medido pelo alfa de Cronbach foi de 0,78 , indicando que a exclusão do item 48 ( $E$ u faço as atividades exigidas nesta disciplina porque elas valem notas), alteraria o alfa para 0,84 . A subescala ficou então composta de 6 itens.

A avaliação do uso de estratégias de aprendizagem constituiu a quarta parte do instrumento, com 19 afirmativas em escala de cinco pontos, variando de "nunca (1)" a "sempre (5)". Os itens foram extraídos do instrumento criado por Accorsi (2005) e, como na subescala de avaliação de instrumentalidade, também foi solicitado aos alunos que levassem em conta a disciplina indicada no instrumento. Um exemplo de item de avaliação de estratégia de superfície é "Eu aprendo repetindo o conteúdo várias vezes para mim mesmo" e, de estratégia de profundidade, "Faço esquemas ou diagramas para me ajudar a entender os textos". O alfa de Cronbach da subescala de avaliação das estratégias de aprendizagem foi de 0,86 para as estratégias de profundidade e 0,62 para as estratégias de superfície.

A intenção de permanecer no curso até a sua conclusão foi avaliada a partir de uma escala na qual os alunos deveriam assinalar de 1 (nenhuma intenção) a 10 (intenção plena). A percepção de desempenho também foi avaliada a partir de uma escala variando de 1 a 10 (fraco, médio e forte).

\section{Procedimento}

Antes da aplicação, o instrumento foi testado com 12 alunos do curso de Arquivologia da mesma instituição, com o objetivo de se verificar a clareza e a compreensão das questões. Foi solicitado aos alunos que anotassem em um formulário, no verso do questionário, as dúvidas e as observações sobre os itens do instrumento. Este procedimento resultou na exclusão de seis questões.

A aplicação da versão final do instrumento para os 143 alunos de Biblioteconomia ocorreu no término do segundo semestre letivo de 2006, durante o horário normal de aula. Antes de iniciar a coleta, a pesquisadora explicou aos alunos o propósito da pesquisa e forneceu as orientações necessárias para o preenchimento do instrumento. Foi destacado o caráter confidencial dos dados e os participantes assinaram um termo de consentimento livre e esclarecido, gastando em média 20 minutos para responder ao questionário. É importante destacar que todas as ações para a coleta de dados foram realizadas com respeito aos direitos do participante.

\section{Resultados}

O presente estudo foi de natureza exploratória e correlacional acerca da motivação de universitários e suas relações com variáveis como a Perspectiva de Tempo Futuro, percepção de instrumentalidade, uso de estratégias de aprendizagem, percepção de desempenho e intenção de permanecer no curso. Para responder aos objetivos propostos, os dados coletados (mediante questionário com itens em escala likert) foram analisados utilizando-se estatística descritiva, correlação e análise de regressão.

Para a avaliação da qualidade da motivação, o desempenho dos 143 participantes foi calculado por meio de média simples, ou seja, diante do diferente número de itens que compuseram cada subescala, optou-se por analisar a média de desempenhos. Observa-se, na Tabela 1, que o desempenho na avaliação da desmotivação e dos tipos mais controlados de motivação extrínseca (por regulação externa e introjetada) foram mais baixos, comparados ao desempenho nos tipos mais autônomos (por regulação identificada e motivação intrínseca). Isto é, os resultados indicaram uma tendência maior para comportamentos com regulação autônoma (Tabela 1). 
Tabela 1. Estatística descritiva dos resultados da avaliação dos tipos de motivação

\begin{tabular}{|l|c|c|c|c|c|}
\hline Variável & Média Simples & Mediana & Mínimo & Máximo & Desvio padrão \\
\hline Desmotivação & 1,41 & 1,00 & 1,00 & 5,80 & 0,73 \\
\hline Regulação Externa & 2,35 & 2,16 & 1,00 & 5,00 & 0,87 \\
\hline Regulação Introjetada & 3,75 & 3,75 & 1,00 & 7,00 & 1,43 \\
\hline Regulação Identificada & 4,79 & 5,00 & 1,00 & 7,00 & 1,39 \\
\hline Motivação Intrínseca & 3,66 & 3,75 & 1,25 & 5,25 & 0,86 \\
\hline
\end{tabular}

Tabela 2. Coeficientes de correlação entre as variáveis do estudo.

\begin{tabular}{|l|c|c|c|c|c|c|}
\hline & $\begin{array}{c}\text { Estratégias } \\
\text { Superfície }\end{array}$ & $\begin{array}{c}\text { Estratégias } \\
\text { Profundidade }\end{array}$ & $\begin{array}{c}\text { Percepção } \\
\text { Desempenho }\end{array}$ & $\begin{array}{c}\text { Intenção de } \\
\text { permanência }\end{array}$ & $\begin{array}{c}\text { Percepção de } \\
\text { instrumentalidade }\end{array}$ & PTF \\
\hline Desmotivação & 0,04 & $-0,17^{*}$ & $-0,29^{* * *}$ & $-0,39^{* * *}$ & $-0,29^{* * *}$ & $-0,13$ \\
\hline $\begin{array}{l}\text { Regulação } \\
\text { Externa }\end{array}$ & 0,08 & $-0,14$ & $-0,22^{* * *}$ & $-0,06$ & $-0,11$ & 0,02 \\
\hline $\begin{array}{l}\text { Regulação } \\
\text { Introjetada }\end{array}$ & $0,34^{* * *}$ & 0,08 & $-0,08$ & $-0,04$ & 0,20 & 0,14 \\
\hline $\begin{array}{l}\text { Regulação } \\
\text { Identificada }\end{array}$ & $0,16^{*}$ & 0,07 & 0,05 & $-0,01$ & $0,33^{* * *}$ & $0,24^{* *}$ \\
\hline $\begin{array}{l}\text { Motivação } \\
\text { Intrínseca }\end{array}$ & 0,06 & $0,21^{*}$ & 0,06 & $0,17^{* * *}$ & $0,32^{* * *}$ & $0,27^{* *}$ \\
\hline
\end{tabular}

${ }^{*} p \leq=0,01$

Os resultados da avaliação do uso de estratégias de aprendizagem (profundidade e superfície) demonstraram médias bem equivalentes - Estratégias de Superfície $(2,55)$ e Estratégias de Profundidade $(2,88)$.

A média de percepção de desempenho foi de 7,27 e a intenção de permanecer no curso até a sua conclusão foi de 9,44 . O desempenho na avaliação da Perspectiva de Tempo Futuro resultou em uma média de 5,29 e desvio padrão 0,67. Quanto à percepção de instrumentalidade, a média obtida pelos participantes foi de 5,47 e o desvio padrão de 0,99.

Para identificar as relações entre as variáveis, utilizamos a Correlação de Pearson. Na Tabela 2, estão demonstrados os coeficientes de correlação entre os tipos de motivação com as estratégias de aprendizagem, percepção de desempenho, percepção de instrumentalidade, Perspectiva de Tempo Futuro e intenção de permanecer no curso.

Apesar de terem sido baixas as correlações encontradas entre os tipos de motivação e as variáveis indicadas, os resultados revelaram que o uso de estratégias de aprendizagem de profundidade (estratégias cognitivas mais elaboradas, como, por exemplo, as paráfrases e resumos) esteve correlacionado de modo positivo e significativo com a motivação intrínseca e, por outro lado, negativo e significativo com a desmotivação. O uso de estratégias de superfície (estratégias cognitivas simples como a prática ou repetição) correlacionou-se positivamente com a motivação extrínseca por regulação introjetada. Descobriu-se correlação negativa entre a desmotivação e a motivação extrínseca por regulação externa com a percepção de desempenho dos alunos. Também foi negativa a correlação entre a intenção de permanecer com a desmotivação. No que se refere à percepção de instrumentalidade e à Perspectiva de Tempo Futuro, foram descobertas correlações positivas entre a percepção de instrumentalidade e a motivação extrínseca por regulação identificada e motivação intrínseca, bem como correlação negativa entre a percepção de instrumentalidade e a desmotivação.

Em outra linha de análise, foram buscadas as comparações entre as médias na avaliação da Perspectiva de Tempo Futuro e a instrumentalidade com as séries dos participantes. A análise de variância (ANOVA) indicou diferenças significativas na avaliação da percepção de instrumentalidade das disciplinas entre as séries, sendo maiores as médias obtidas pelos alunos da $1^{\mathrm{a}}$ e $2^{\mathrm{a}}$ séries, $\mathrm{F}(3,142)=2,99$, em 
nível de $p=0,03$, como mostra a Tabela 3 . O teste Tukey (HSD) revelou que as médias dos participantes da $1^{\text {a }}$ série foram significativamente maiores do que as obtidas pelos alunos da $4^{\text {a }}$ série (Tabela 3 ).

Tabela 3. Resultados do Teste Tukey para as diferenças entre o desempenho na percepção de instrumentalidade de acordo com a série dos participantes.

\begin{tabular}{|l|c|c|c|c|}
\hline Séries & $\mathbf{( 1 )}$ & $\mathbf{( 2 )}$ & $\mathbf{( 3 )}$ & $\mathbf{( 4 )}$ \\
\hline & $\mathrm{M}=5,92$ & $\mathrm{M}=5,82$ & $\mathrm{M}=5,53$ & $\mathrm{M}=5.02$ \\
\hline $1(1)$ & - & 0,97 & 0,34 & 0,03 \\
\hline $2(2)$ & & - & 0,64 & 0,10 \\
\hline $3(3)$ & & & - & $0,56^{*}$ \\
\hline $4(4)$ & & & & - \\
\hline
\end{tabular}

${ }^{*} \mathrm{p} \leq 0,05$

Um importante objetivo da presente pesquisa foi verificar se a Perspectiva de Tempo Futuro dos alunos seria variável preditiva da motivação, da intenção de permanecer no curso, da percepção de desempenho e do uso de estratégias de aprendizagem. Esta verificação foi realizada por meio da análise de regressão, que permitiu aferir a contribuição relativa de elementos contidos no instrumento para explicar a variância do desempenho dos participantes nas varáveis consideradas dependentes (motivação, percepção de instrumentalidade, uso de estratégias de aprendizagem, intenção de permanecer no curso e percepção de desempenho). Uma síntese dos resultados pode ser vista na Tabela 4 .

Tabela 4. Resultados positivos da análise de regressão entre a Perspectiva de Tempo Futuro e as variáveis motivacionais de percepção de instrumentalidade e do uso de estratégias de profundidade.

\begin{tabular}{|l|c|c|c|}
\hline Variáveis Dependentes & $\beta$ & $p$ & $R^{2}$ \\
\hline $\begin{array}{l}\text { Motivação extrínseca por regulação } \\
\text { identificada }\end{array}$ & 0,24 & 0,002 & 0,06 \\
\hline Motivação Intrínseca & 0,27 & 0,000 & 0,07 \\
\hline Percepção de Instrumentalidade & 0,35 & 0,000 & 0,12 \\
\hline Uso de estratégias de profundidade & 0,18 & 0,02 & 0,03 \\
\hline
\end{tabular}

A Perspectiva de Tempo Futuro foi preditiva para as formas autônomas de motivação, da percepção de instrumentalidade das atividades do presente para o alcance de metas colocadas no futuro e do uso de estratégias de profundidade nas tarefas de aprendizagem. A Perspectiva de Tempo Futuro como preditor único das variáveis analisadas explicou uma pequena porção da variância dos dados, com exceção da percepção de instrumentalidade. Não foram encontrados valores significativos de predição em relação às demais variáveis investigadas.

\section{Discussão}

Os resultados referentes às orientações motivacionais dos alunos demonstraram uma tendência para a motivação extrínseca por regulação introjetada, identificada e para motivação intrínseca. Esse pode ser um indicador de que os alunos percebem a regulação do seu comportamento de forma mais autônoma do que controlada, visto que, na regulação introjetada, apesar do locus de causalidade ainda ser externo, há um caráter levemente autônomo para a apresentação do comportamento. Na motivação extrínseca por regulação identificada, o nível de autonomia é mais alto, havendo uma identificação e empatia com a atividade e o aluno reconhece sua importância e a valoriza pessoalmente. A motivação intrínseca é o nível mais desejável de regulação autônoma, situação na qual a escolha e a realização de determinada atividade acontece pelo interesse inerente a ela. Esse é o fenômeno considerado o melhor representante do potencial positivo da natureza humana, essencial para o desenvolvimento cognitivo, social e bem-estar pessoal (Ryan \& Deci, 2000b). O desempenho na avaliação da motivação intrínseca foi um resultado bastante positivo, uma vez que comportamentos com esse tipo de orientação não são tão comuns no contexto acadêmico.

Csikszentmihalyi (1992) evidenciou que as experiências de aprendizagem oferecidas pela escola geralmente são motivadas extrinsecamente, sendo que os alunos se sentem aliviados quando concluem ou se evadem de seus cursos. Assim, é muito importante que os professores do curso em questão valorizem e fortaleçam essas orientações intrínsecas dos alunos com atividades que representem desafios ótimos e que estimulem ainda mais o comprometimento com o processo de aprendizagem, visando sempre à promoção de comportamentos autodeterminados.

Em relação aos dois tipos de motivação extrínseca que se sobressaíram na avaliação da motivação deste grupo de universitários, Ryan e Deci (2000b) afirmam que, embora revestida de sentimentos de ansiedade, a regulação introjetada tem sido positivamente relacionada ao esforço. De modo semelhante, em estudos citados pelos autores, a regulação identificada foi associada a interesse, persistência e maior dedicação de esforços no desenvolvimento das atividades escolares. Pode-se, assim, inferir que a motivação acadêmica na amostra estudada tem uma inclinação a comportamentos regulados internamente, levando-se a presumir que esses alunos acreditam serem capazes de realizar uma atividade por vontade própria e não apenas por pressões externas, têm sentimentos de liberdade e de responsabilidade pelas suas metas e ações e visualizam a possibilidade de escolha no decorrer das atividades acadêmicas. As regras e pressões do contexto do próprio curso, possivelmente, 
são endossadas ou coincidem com os valores e regulações pessoais dos alunos.

Em relação ao emprego de estratégias de aprendizagem, os participantes obtiveram desempenho semelhante na avaliação do uso de estratégias de profundidade e de superfície. Pode ser que essa questão esteja relacionada ao desconhecimento das estratégias de aprendizagem ou à falta de compreensão sobre como e quando utilizá-las. Boruchovitch (1999) já havia destacado que o uso de estratégias requer uma compreensão de como estas funcionam. Isto é, além de conhecer um bom repertório de estratégias, os alunos precisam saber usá-las de maneira eficaz, selecionando-as de acordo com as necessidades de cada atividade.

Em relação à Perspectiva de Tempo Futuro, os resultados permitem inferir que os alunos participantes da pesquisa estabelecem e valorizam as metas de longo prazo. Esse pode ser um indicativo de motivação, uma vez que, teoricamente, as concepções de futuro influenciam positivamente a persistência, o esforço, a valorização das atividades e, consequentemente, o desempenho acadêmico. Vários estudos empíricos, como, por exemplo, De Volder e Lens (1982), Miller, Debacker e Greene (1999) e Van Calster, Lens e Nuttin (1987), evidenciaram que quanto maior o valor atribuído às metas futuras, maior será a motivação para o desenvolvimento das atividades do presente.

A avaliação da percepção de instrumentalidade sugeriu que os alunos conseguem perceber a utilidade das atividades do presente para a sua atuação futura. Esse resultado pode ser considerado importante, já que essa percepção contribui para a valorização das atividades e, por conseguinte, promove o envolvimento e a aplicação de esforço. Para Raynor (1981 citado por Husman \& cols. 2004), a valorização de uma atividade determina a qualidade e a quantidade de esforço empenhado na sua realização. Os alunos que percebem a utilidade das atividades acadêmicas são mais entusiasmados, dedicam maior esforço para dominar todas as tarefas e têm comportamentos mais motivados do que os alunos que as veem apenas para treinamento. Isso foi confirmado por Simons e cols. (2004) em pesquisa realizada com alunos de diferentes cursos universitários.

Contrariando as expectativas iniciais, uma importante descoberta foi que, conforme os alunos avançam nas séries, a percepção de instrumentalidade das atividades exigidas pelas disciplinas do curso diminui. A diferença significativa apareceu entre os alunos da $1^{a}$ e $4^{a}$ série, indicando que os da $1^{\text {a }}$ série percebem as atividades acadêmicas com maior instrumentalidade para o seu futuro profissional. Stipek (1998), apesar de analisar a motivação de alunos do Ensino Fundamental, afirma que a complexidade dos problemas motivacionais tende a aumentar conforme os alunos avançam nas séries. Nessa mesma direção, especificamente em relação a universitários, Jacobs e Newstead (2000) também evidenciaram que o grau de motivação pode variar no decorrer de um curso superior, tendo em vista que a percepção da relevância dos conteúdos e habilidades modifica-se de acordo com as diferentes séries e disciplinas.
Outra possível explicação é que a diminuição da percepção de instrumentalidade, encontrada na presente pesquisa, pode estar relacionada com o tipo de disciplina ofertado nessas séries. Enquanto nas duas primeiras séries do Curso de Biblioteconomia há um agrupamento maior de disciplinas que são especificamente técnicas (catalogação, classificação, normalização, análise documentária e outras) e de fácil associação com a atuação prática, nas séries subsequentes são ofertadas disciplinas com conteúdos mais abrangentes (planejamento, gestão, mediação, recursos informacionais, políticas culturais, ciência e tecnologia, inovação, desenvolvimento de projetos e outros). Estas últimas exigem maior domínio e reflexão para a articulação com as ações práticas da atuação profissional futura, justificando, assim, a diminuição do valor instrumental. Dessa forma, a percepção de instrumentalidade necessita ser estimulada, ou seja, compete ao professor mostrar o valor das atividades do presente para o alcance das metas futuras.

$\mathrm{Na}$ relação entre a motivação e o uso de estratégias de aprendizagem, evidenciou-se que as estratégias de profundidade estão relacionadas com a motivação intrínseca e as estratégias de superfície com os tipos de motivação menos autônomos. Esse resultado está alinhado com várias pesquisas (Accorsi, 2005; Cardoso, 2002; Machado, 2005; entre outras) que encontraram relações significativas entre as estratégias de profundidade e a meta aprender, cujas características têm algumas similaridades com a motivação intrínseca.

Em relação à percepção de desempenho, os resultados indicaram bom nível de desempenho acadêmico na amostra pesquisada, correlacionando-se de modo negativo com a desmotivação e a regulação externa. Estes resultados são compreensíveis, já que para estes dois tipos de envolvimento são características a desvalorização, a falta de percepção de controle e a submissão às regras ou exigências externas, levando o aluno a se sentir totalmente alheio ao seu próprio desempenho. É importante mencionar que a opção de avaliar o desempenho a partir da percepção dos alunos, e não por notas, é apoiada por Basow e Medecalf (1988), Covington e Omelich (1981), entre outros que, em todas as suas investigações, encontraram uma alta correlação entre a percepção de desempenho e o desempenho real dos alunos.

A intenção de permanecer no curso até a sua conclusão foi apontada pela grande maioria dos participantes, mostrando que estes são persistentes na busca de suas metas. A persistência é um dos cinco indicadores comportamentais da motivação mencionado por Stipek (1998) e refere-se à extensão de tempo que o aluno dedica a uma atividade, independente das dificuldades encontradas. Vale salientar que esse resultado vem ao encontro da atual realidade do curso, em que se observa um índice de evasão de alunos bastante reduzido.

A relação positiva entre a percepção de instrumentalidade e Perspectiva de Tempo Futuro com a motivação, especificamente, com os tipos mais autônomos de regulação (regulação identificada e motivação intrínseca) é uma 
confirmação do que tem sido abundantemente relatado na literatura. De Volder e Lens (1982), Lens, Simons e Dewite (2002), Simons e cols. (2004) evidenciaram, a partir de seus estudos, que os alunos mais motivados conferem maior valor às metas futuras e percebem suas atividades como instrumentais para atingi-las.

Finalmente, os resultados indicaram um valor de predição da Perspectiva de Tempo Futuro para a percepção de instrumentalidade, para a motivação extrínseca por regulação identificada e para motivação intrínseca. Pode-se concluir que, para os alunos participantes deste estudo, o estabelecimento de metas futuras pode prever positivamente a valorização das atividades exigidas no presente e, consequentemente, a motivação autônoma. Nesse sentido, é importante destacar que a Perspectiva de Tempo Futuro teve maior valor preditivo em relação à percepção de instrumentalidade, revelando que os alunos percebem o valor das atividades acadêmicas do presente para a realização de metas futuras.

O valor de utilidade é um dos componentes da valorização, na perspectiva da Teoria Expectativa-Valor, e consiste na percepção da utilidade de uma atividade para a realização de planos futuros. Essa constatação permite-nos refletir sobre a questão do controle externo, pois se considerarmos que um aluno realiza uma tarefa pelo seu valor de utilidade, logo, uma orientação externa, será que este tipo de regulação do comportamento prejudicaria as orientações motivacionais intrínsecas? Os resultados desta pesquisa parecem revelar que não houve prejuízos para a motivação intrínseca, tendo em vista que o desempenho dos alunos foi maior na avaliação dos tipos mais autônomos de motivação (motivação por regulação introjetada, por regulação identificada e motivação intrínseca). Dessa forma, os resultados encontrados são coerentes e confirmam a alegação de Husman e Lens (1999) de que a Perspectiva de Tempo Futuro e a percepção de instrumentalidade não prejudicam a motivação intrínseca, desde que o grau de regulação do comportamento seja interno, ou seja, integrado ao self.

A Perspectiva de Tempo Futuro também se mostrou preditora do uso de estratégias de aprendizagem de profundidade e, mesmo sendo uma predição moderada, é um indicativo da relevância da fixação de metas futuras para o envolvimento, aproveitamento e bom desempenho dos alunos nas atividades acadêmicas. Por outro lado, a percepção de desempenho e a intenção de permanecer no curso não demonstraram dependência em relação à Perspectiva de Tempo Futuro. O resultado da falta de relação entre Perspectiva de Tempo Futuro e a intenção de permanência no curso foi inesperado, pois se acreditava que a inserção futura no mercado de trabalho poderia ser uma das variáveis preditivas da persistência no curso. Na amostra estudada, portanto, não há uma relação direta entre a intenção de permanência e as metas profissionais futuras.

Outro resultado revelado pela análise de regressão foi que Perspectiva de Tempo Futuro não apareceu como a única preditora das variáveis analisadas, já que explicou uma pequena proporção da variância dos dados. Isto quer dizer que existem outros elementos, não incluídos no estudo, que funcionam como preditores das orientações motivacionais. Esse resultado está coerente com constatação de Creten, Lens e Simons (2001 citado por Lens e cols., 2002; Simons e cols. 2004) de que a percepção de instrumentalidade tem uma contribuição importante, mas não pode ser considerada como condição suficiente para o desenvolvimento da motivação dos alunos.

Assim, fica evidente que, para promover a motivação no contexto escolar, outros aspectos além da Perspectiva de Tempo Futuro e da instrumentalidade são necessários, como, por exemplo, a satisfação das necessidades dos alunos por autonomia, competência e pertencimento, que integram a subteoria das Necessidades Básicas da Teoria da Autodeterminação. Vale ressaltar que, no contexto do curso de Biblioteconomia, a satisfação das necessidades psicológicas básicas pode ser uma das variáveis que tem contribuído para a permanência dos alunos no curso, necessitando de novos estudos para a constatação desta hipótese.

\section{Considerações Finais}

Esta pesquisa trouxe importantes contribuições, uma vez que os resultados mostraram vários aspectos que podem estar envolvidos na motivação acadêmica, ampliando a compreensão das orientações motivacionais de alunos de cursos superiores.

Além disso, é possível visualizar algumas implicações educacionais. A primeira refere-se à constatação de que a Perspectiva de Tempo Futuro e a instrumentalidade têm uma relação significativa com a motivação extrínseca por regulação identificada e com a motivação intrínseca, revelando, assim, a importância do professor articular em sala de aula as atividades do presente com a atuação profissional futura. Contudo, o fato da Perspectiva de Tempo Futuro não se configurar como única variável que explica as orientações motivacionais merece uma atenção especial, já que esse resultado evidencia a necessidade do uso de diferentes estratégias para promover e manter a motivação no contexto acadêmico.

Outro aspecto a se considerar diz respeito à percepção de instrumentalidade com regulação interna. Essa combinação pode ser exemplificada com aquele aluno que, além de perceber as atividades do presente como úteis para a sua atuação profissional futura, visualiza o valor destas para o seu crescimento pessoal. Dessa forma, é importante estimular nos alunos o planejamento e a adoção de metas futuras de maneira autônoma, possibilitando que estes as valorizem pessoalmente.

No que se refere às estratégias de aprendizagem, cabe ao professor evidenciar a necessidade do uso adequado de estratégias no decorrer da vida acadêmica, levando o aluno a dominar os diferentes tipos de estratégias e a refletir sobre a maneira mais eficaz de como utilizá-las, considerando que, na maioria das vezes, muitos alunos chegam aos 
seus cursos superiores sem mesmo conhecer essas estratégias de aprendizagem.

\section{Referências}

Accorsi, D. M. P. (2005). Envolvimento cognitivo de universitários em relação à motivação contextualizada. Dissertação de Mestrado, Universidade Estadual de Londrina, Londrina, Paraná.

Basow, S. A., \& Medcalf, K. L. (1988). Academic achievement and attributions among college students: effects of gender and sex typing. Sex Roles, (9/10), 555-567.

Boruchovitch, E. (1999). Estratégias de aprendizagem e desempenho escolar: considerações para a prática educacional. Psicologia: Reflexão e Crítica, 22(2), 361-376.

Boruchovitch, E., Bzuneck, J. A. (2010). Motivação para aprender no Brasil: estado da arte e caminhos futuros. Em E. Boruchovitch, J. A. Bzuneck \& S. É. R. Guimarães, (Orgs.), Motivação para aprender: aplicações no contexto educativo (pp 231-254). Petrópolis, RJ: Vozes.

Cardoso, L. R. (2002). Uso de estratégias de aprendizagem e suas relações com metas de realização: um estudo no ensino superior. Dissertação de Mestrado, Universidade Estadual de Londrina, Londrina-PR.

Covington, M. V., \& Omelich, C. L. (1981). As failure mount: affective and cognitive consequences of ability demotion in the classroom. Journal of Educational Psychology, (73), 796-808.

Csikszentmihalyi, M. (1992). A psicologia da felicidade. São Paulo: Saraiva.

De Volder, M. L., \& Lens, W. (1982). Academic achievement and future time perspective as a cognitive-motivational concept. Journal of Personality and Social Psychology, 42(3), 566-571.

Deci, E. L., \& Ryan, R, M. (2000). The "what" and "why" of goal pursuits: human needs and the self-determination of behavior. Psychological Inquiry, 11(4), 227-268.

Deci, E. L., \& Ryan, R, M. (2008). Self-determination theory: A macrotheory of human motivation, development and health. Canadian Psychology, 49, 182-185.

Guimarães, S. É. R., \& Bzuneck, J. A. (2008). Propriedades psicométricas de um instrumento para avaliação da motivação de universitários. Ciências \& Cognição, 13(1), 101-103.

Husman, J., \& Lens, W. (1999). The role of the future in student motivation. Educational Psychologist, 34, 113-125.

Husman, J., Derryberry, W. P., Crowson, H. M., \& Lomax, R. (2004). Instrumentality, task value, and intrinsic motivation: making sense of their independent interdependence. Contemporary Educational Psychology, (29), 63-76.

Jacobs, P. A., \& Newstead, S. E. (2000). The nature and development of student motivation. British Journal of Educational Psychology, 70(2), 243-254.

Kauffman, D. F., \& Husman, J. (2004). Effects of time perspective on student motivation: introduction to a special issue. Educational Psychology Review, 16(1), 1-7.

Lens, W., Simons, J., \& Dewitte, S. (2002). The role of students's future time perspective and instrumentality percepctions for study motivation and self-regulation. Em F. Pajares \& T. Urdan. Academic motivation of adolescents (pp.221-245). Greenwich: IAP.

Machado, O. A. (2005). Evasão de alunos de cursos superiores: fatores motivacionais e de contexto. Dissertação de Mestrado Universidade Estadual de Londrina, Londrina, Paraná.

Malka, A., \& Covington, M.V. (2005). Perceiving school performance as instrumental to future goal attainment: efects on graded performance. Contemporary Educational Psychology, 30, 60-80.

Mclnerney, D. M. (2004). A discussion of future time perspective. Educational. Psychology Review, 16(2), 141-151.

Miller, R. B., \& Brickman, S. J. (2004). A model of future-oriented motivation and self-regulation. Educational Psychology Review, 16(1), 9-36.

Miller, R. B., Debacker, T. K., \& Greene, B. A. (1999). Perceived instrumentality and academics: the link to task valuing. Journal of Instructional Psychology, 26(4), 250-259.

Phalet, K., Andriessen, I., \& Lens, W. (2004). How future goals enhance motivation and learning in multicultural classrooms. Educational Psychology Review, 16(1), 59-89.

Reeve, J., Deci, E. L., \& Ryan, R. M. (2004). Self-determination theory: a dialectical framework for understanding sociocultural influences on student motivation. Em D. M. Mclnerney \& S. Van Etten (Eds.), Big theories revisited (pp.31-60). Greenwich: Information Age Publishing.

Ryan, R. M., \& Deci, E. L. (2000a). Intrinsic and extrinsic motivations: classic definitions and new directions. Contemporary Educational Psychology, (25), 54-67.

Ryan, R. M., \& Deci, E. L. (2000b). Self-determination theory and the facilitation of intrinsic motivation, social development and wellbeing. American Psychologist, 55(1), 68-78.

Shell, D. F., \& Husman, J. (2001). The multivariate dimensionality of personal control and future time perspective beliefs in achievement and self-regulation. Contemporary Educational Psychology, 26, 481-506. 
Simons, J., Dewitte, S., \& Lens, W. (2004). The role of different types of instrumentality in motivation, study strategies, and performance: know why you learn, so you`ll know what you learn. British Journal Educational Psychology, (74), 343-360.

Simons, J., Vansteenkiste, M., Lens, W., \& Lacante, M. (2004). Placing motivation and Future Time Perspective theory in a temporal perspective. Educational Psychology Review, 16(2), 121-139.

Stipek, D. J. (1998). Motivation to learn: from theory to practice. Englewood Cliffs, NJ: Preintice Hall.

Vallerand, R. J., Pelletier, L. G., Blais, M. R., Briere, N. M., Senecal, C., \& Vallieres, E. F. (2002). The academic motivation scale: a measure of intrinsic, extrinsic, and amotivation in education Educational and Psychological Measurement, 52, 1003-1017.

Van Calster, K., Lens, W., \& Nuttin, J. R. (1987). Affective attitude toward the personal future: Impact on motivation in high school boys. American Journal Psychologist, 100(1), 1-13.

Vansteenkiste, M., Lens, W., \& Deci, E. L. (2006). Intrinsic versus extrinsic goal contents in self-determination theory: another look at the quality of academic motivation. Educational Psychologist, 41(1), 19-31.

\section{Sobre as autoras}

Adriana Rosecler Alcará (adrianaalcara@sercomtel.com.br) Universidade Estadual de Londrina, Londrina - PR

Sueli Édi Rufini Guimarães (sueli.rufini@ pesquisador.cnpq.br) Universidade Estadual de Londrina, Londrina - PR

\section{Correspondência}

